S. Itatsu and H. Kaneta

Nagoya Math. J.

Vol. 79 (1980), 23-32

\title{
SPECTRAL PROPERTIES OF FIRST ORDER ORDINARY DIFFERENTIAL OPERATORS WITH SHORT RANGE POTENTIALS
}

\author{
S. ITATSU AND H. KANETA
}

\section{$\S 1$. Introduction and main theorem}

The purpose of the present paper is to give a complete proof of the theorem which will be used in a paper of the second author [4].

We will discuss certain spectral properties of selfadjoint ordinary differential operators of the form $i A(d / d x)+V$ acting in $L^{2}(R)_{n}=\sum \oplus L^{2}(R)$, where $A$ is a real diagonal constant matrix and $V$ an Hermitian matrix valued function on $\boldsymbol{R}$ which satisfies some conditions to be stated in the sequel.

According to [1, p. 156] a function $v$ in $L_{10 c}^{2}(R)$ is said to belong to the class $S R$ if, for some $\varepsilon>0$, the multiplication map: $u(x) \rightarrow(1+$ $|x|)^{1+\varepsilon} v(x) u(x)$ is a compact operator from the Sobolev space $H_{1}(R)$ into $L^{2}(\boldsymbol{R})$ (the square integrable functions on $\boldsymbol{R}$ ). For a selfadjoint operator $L$ in a Hilbert space $\mathfrak{S}_{\mathrm{g}}$, let $L_{\mathrm{p}}, L_{\mathrm{c}}$ and $L_{\mathrm{ac}}$ stand, respectively, for the restriction of $L$ to the subspace $\mathfrak{S}_{\mathrm{p}}$ spanned by all eigenvectors, $\mathfrak{F}_{\mathfrak{p}}^{\perp}$ (the orthogonal complement of $\mathfrak{S}_{\mathrm{p}}$ ) and the absolutely continuous subspace $\mathfrak{F}_{\mathrm{ac}}\left([5]\right.$, p. 516). Thus we have $L=L_{\mathrm{p}} \oplus L_{\mathrm{c}}$ and $L_{\mathrm{c}} \supset L_{\mathrm{ac}}$. Let $A$ be a real diagonal $n \times n$-matrix with $(j, j)$-component $a_{j}$ and $V$ an Hermitian $n \times n$ matrix valued function on $\boldsymbol{R}$ with the $(j, k)$-component $V_{j k}$ in $L_{\mathrm{loc}}^{2}(R)$. As will be shown in Lemma 1 , the symmetric operator $\dot{L}=i A(d / d x)+V$ with domain $C_{o}^{\infty}(R)_{n}=\sum \oplus C_{o}^{\infty}(R)$ is essentially selfadjoint in the Hilbert space $L^{2}(\boldsymbol{R})_{n}=\sum \oplus L^{2}(\boldsymbol{R})$, we denote the selfadjoint extension of $\dot{L}$ by $L$. Then our main result is the following.

TheOREM. (i) Assume that $a_{1} \cdots a_{n} \neq 0$. If each matrix element $V_{j k}$ of $V$ belongs to the class $S R$, then $L=L_{\mathrm{ac}}$. Under the additional assumption that for some $\varepsilon>0$ and $0<\theta<1 / 2$ each $V_{j k}$ satisfies

Received September 8, 1978. 


$$
\sup _{x \in R}\left[(1+|x|)^{2+2 s} \int_{|x-y| \leqslant 1}\left|V_{j k}(y)\right|^{2}|y-x|^{1-2 \theta} d y\right]<\infty,
$$

$L_{\mathrm{ac}}$ is unitarily equivalent to the selfadjoint multiplication operator $M$ in $L^{2}(R)_{n}$ defined by $M f(\lambda)=\lambda f(\lambda)$. Note that the condition $\left(^{*}\right)$ is satisfied if $V_{j k}(x)=O\left(|x|^{-1-\varepsilon}\right)$ as $|x| \rightarrow \infty$.

(ii) Assume that $a_{1}=\cdots=a_{m}=0$ and $a_{m+1} \cdots a_{n} \neq 0$ for some $0<$ $m<n$ and that

$$
\left\{\begin{array}{c}
V_{j k}=0 \quad \text { for } j, k=1, \cdots, m, \\
V_{j k} \text { is bounded for } j=1, \cdots, m \text { and } k=m+1, \cdots, n, \\
V_{j k} \text { and } W_{j k}=\sum_{1 \leqslant \ell \leqslant m} V_{j \ell} V_{\ell k} \text { are of the class } S R \text { for } j, k \\
=m+1, \cdots, n .
\end{array}\right.
$$

Then $L$ has no eigenvalues differing from zero and $L_{\mathrm{c}}=L_{\mathrm{ac}}$. In addition, if each $V_{j k}$ belongs to $C^{1}(R)$ and satisfies

$$
V_{j k}(x)=O\left(|x|^{-1-s}\right) \quad \text { as }|x| \longrightarrow \infty
$$

for some $\varepsilon>0$, then $L_{\mathrm{c}}$ is unitarily equivalent to the selfadjoint multiplication operator $M$ in $L^{2}(\boldsymbol{R})_{n-m}$ defined by $M f(\lambda)=\lambda f(\lambda)$.

In $\S 3$ a sufficient condition for $L$ to have no eigenvalues will be found.

\section{§2. Proof of the theorem}

We proceed as Agmon [1]. To begin with, we explain our notations. The real and complex numbers will be denoted by $R$ and $C$ respectively. As usual, $C_{ \pm}=\{z \in C: \pm \operatorname{Im} z>0\}$ and $R^{*}=R \backslash\{0\}$.

$$
L_{\mathrm{loc}}^{p}(R)=\left\{u(x): \int_{K}|u(x)|^{p} d x<\infty \text { for any compact set } K \text { in } R\right\} .
$$

$L^{2}(R)=$ the square integrable functions with the usual norm \|\| . For real $s$,

$$
\begin{aligned}
L^{2, s}(R)= & \left\{u(x):\left(1+x^{2}\right)^{s / 2} u \in L^{2}(R)\right\} \text { with the norm }\|\|_{0, s}: \\
& \|u\|_{0, s}=\left\|\left(1+x^{2}\right)^{s / 2} u\right\| .
\end{aligned}
$$

For any integer $m \geq 0$ and real $s$, we define the weighted Sobolev space $H_{m, s}(R)$ by

$$
\begin{gathered}
H_{m, s}(R)=\left\{u(x): D^{\alpha} u \in L^{2, s}(R), 0 \leqslant m\right\} \text { with the norm }\|\|_{m, s}: \\
\|u\|_{m, s}=\left(\sum_{0 \leqslant \alpha \leqslant m}\left\|D^{\alpha} u\right\|_{0, s}^{2}\right)^{1 / 2}, \text { where } D=-i \frac{d}{d x} .
\end{gathered}
$$


For real $m$, the Sobolev space $H_{m}(R)$ of order $m$ is defined as the completion of $C_{o}^{\infty}(R)$ under the norm

$$
\|u\|_{m}=\int|\hat{u}(\lambda)|^{2}\left(1+\lambda^{2}\right)^{m} d \lambda .
$$

Here $\hat{u}$ stands for the Fourier transform of $u$, namely,

$$
\hat{u}(\lambda)=(2 \pi)^{-1 / 2} \int u(x) e^{-i x \lambda} d x .
$$

Thus $H_{m, 0}(\boldsymbol{R})=H_{m}(\boldsymbol{R})$ for non-negative integer $m$. The continuous functions and continuously differentiable functions on $R$ will be denoted by $C(R)$ and $C^{1}(R)$ respectively. For any $0<\theta<1$ and real $s$ we denote by $C^{\theta, s}(R)$ the continuous functions such that

$$
\|\left.|u|\right|_{\theta, s}=\sup _{x \in \boldsymbol{R}}(1+|x|)^{s}|u(x)|+\sup _{\substack{x, y \\ 0<|x-y|<1}}\left[(1+|x|)^{s} \frac{|u(x)-u(y)|}{|x-y|^{\theta}}\right]<\infty .
$$

$\boldsymbol{C}^{n}$-valued functions on $\boldsymbol{R}$ whose components lie in $L^{2}(\boldsymbol{R})$, for example, will be denoted by $L^{2}(R)_{n}$.

Finally,

$A$ : a real diagonal matrix with the $(j, j)$-component $a_{j}$.

$V:$ an Hermitian matrix valued function on $\boldsymbol{R}$ whose $(j, k)$-component is $V_{j k}$.

$\tilde{V}:$ an Hermitian matrix valued function on $\boldsymbol{R}$ whose $(j, k)$-component is $V_{j k}(m<j, k \leqslant n)$.

$W$ : an Hermitian matrix valued function on $\boldsymbol{R}$ whose $(j, k)$-component is $W_{j k}=\sum_{1 \leqslant \ell \leqslant m} V_{j \ell} V_{\ell k}(m<j, k \leqslant n)$.

$D_{L}$ : the domain of the operator $L=i A(d / d x)+V$.

Lemma 1. The operator $\dot{L}=i A(d / d x)+V$ with domain $C_{o}^{\infty}(R)_{n}$ is essentially selfadjoint in $L^{2}(\boldsymbol{R})_{n}$.

Proof. Recall that $V_{j k} \in L_{\mathrm{loc}}^{2}(\boldsymbol{R})$. Obviously $\dot{L}$ is symmetric. Assume first that the diagonal matrix is non-degenerate. It remains only to show that the range of $\dot{L}-z$ is dense for any $z \in C_{ \pm}$. To this end, suppose that a $g \in L^{2}(R)_{n}$ satisfies

$$
((\dot{L}-z) f, g)=0 \quad \text { for any } f \in C_{o}^{\infty}(R)_{n} .
$$

Since $V_{j k} \in L_{\mathrm{loc}}^{2}(R)$, (1) implies that $g$ is absolutely continuous and that

$$
i A g^{\prime}+(V-z) g=0 \text {. }
$$


Thus it follows easily that

$$
(A g(x), g(x))^{\prime}=-2 \operatorname{Im} z(g(x), g(x)) .
$$

Since a monotone function in $L^{1}(R)$ is zero, the function $(A g(x), g(x))$ is zero. Now from (3) it follows that $g=0$. Next assume that $a_{1}=\cdots=$ $a_{m}=0$ and $a_{m+1} \cdots a_{n} \neq 0$. Then (1) implies that components $g_{j}(m<j$ $\leqslant n$ ) are absolutely continuous. The rest of the proof is the same as that in the case where $\operatorname{det} A \neq 0$.

Q.E.D.

Remark. The domain $D_{L}$ is $H_{1}(\boldsymbol{R})_{n}$ in the case (i) and $L^{2}(\boldsymbol{R})_{m} \oplus H_{1}(\boldsymbol{R})_{n-m}$ in the case (ii) of our theorem. In order to verify this, recalling the theorem 4.3 of $[5$, p. 287], it suffices to show that there exist some constants $0 \leqslant a$ and $0 \leqslant b<1$ such that

$$
\|v f\|^{2} \leqslant a^{2}\|f\|^{2}+b^{2}\|f\|_{1}^{2}
$$

for a function $v$ belonging to class $S R$ and for any $f \in H_{1}(R)$. To this end, note first that the following inequality holds for some constant $c$.

$$
\left\|(1+|x|)^{1+\varepsilon} v f\right\|^{2} \leqslant c\|f\|_{1}^{2} .
$$

Hence there exists positive constant $r$ such that

$$
\int_{|x| \geqslant r}|v f|^{2} d x \leqslant\|f\|_{1}^{2} / 4
$$

Since $\|f\|_{\infty} \leqslant c\|f\|_{1}^{2}$ for some constant, taking $N$ large enough, we have

$$
\int_{|x|<r}|v f|^{2} d x=\left(\int_{|x|<r,|v| \leqslant N}+\int_{|x|<r,|v|>N}\right)|v f|^{2} d x \leqslant N^{2}\|f\|^{2}+\|f\|_{1}^{2} / 4 .
$$

2.1. Eigenvalues. The following lemma, together with Proposition 3 in $\S 3$, implies that $L$ has no eigenvalues in the case (i) and that $L$ has no eigenvalues differing from zero in the case (ii).

LEMMA 2. If $v$ belongs to the class $S R$, then $v$ is integrable.

Proof. Assume that for a positive $\varepsilon$ the map $u \rightarrow(1+|x|)^{1+\varepsilon} v u$ is a compact operator from $H_{1}(R)$ into $L^{2}(R)$. Then $(1+|x|)^{1+\varepsilon}|v||u|^{2}$ is integrable for any $u \in H_{1}(R)$, in particular, for $u=\left(1+x^{2}\right)^{-(1+s) / 4}$. Q.E.D.

2.2. The limiting absorbtion principle.

Case (i). Let $R_{o}(z)$ be the resolvent $(i A(d / d x)-z)^{-1}$ for $z \in C_{ \pm}$. We note that the theorem 4.1 of [1] holds for $R_{0}(z)$, hence the boundary value 
$R_{o}^{ \pm}(\lambda)$ is a well defined bounded operator in $B\left(L^{2, s}(\boldsymbol{R})_{n}, H_{1,-s}(\boldsymbol{R})_{n}\right)$ for any $s>1 / 2$.

Definition. A function $u \in H_{1}^{\text {loc }}(R)_{n}$ will be called a $\lambda$-outgoing function (resp. $\lambda$-incoming function) if for $\lambda \in R$ the relation holds:

$$
u=R_{o}^{+}(\lambda) f \quad\left(\text { resp. } u=R_{o}^{-}(\lambda) f\right) \text { for some } f \in L^{2, s}(R)_{n}
$$

with some $s>1 / 2$. Among several steps to prove the limiting absorption principle (cf. Theorem 4.2, [1]), Lemma 4.2 of [1] is the only one whose proof needs new idea. A difficulty arises because $A$ is not necessarily definite. Therefore, we confine ourselves to the proof of the following

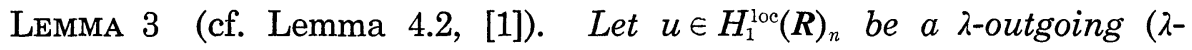
incoming) function satisfying a differential equation in the distribution sense:

$$
\left(i A \frac{d}{d x}+V-\lambda\right) u=0
$$

where the matrix element of $V$ are of class $S R$. Then $u$ belongs to $H_{1, s}(R)_{n}$ for all real $s$.

Proof. We shall prove the lemma for $u$ outgoing, the proof for $u$ incoming is similar. By the assumption, $u=R_{o}^{+}(\lambda) f$ for some $f \in L^{2, s_{o}}(\boldsymbol{R})_{n}$, $s_{o}>1 / 2$. This implies

$$
\begin{aligned}
u_{j}(x) & =i a_{j}^{-1} \int_{x}^{\infty} e^{-i a_{j}^{-1}(x-y) \lambda} f_{j}(y) d y \quad \text { for } j \in J_{+}=\left\{j: a_{j}>0\right\} \\
& =-i a_{j}^{-1} \int_{-\infty}^{x} e^{-i a_{j}^{-1}(x-y) \lambda} f_{j}(y) d y \quad \text { for } j \in J_{-}=\left\{j: a_{j}<0\right\} .
\end{aligned}
$$

Since $f$ is integrable, it follows that $u_{j}(\infty)=0$ (resp. $u_{j}(-\infty)=0$ ) for $j \epsilon$ $J_{+}$(resp. $j \in J_{-}$) and that $u$ is absolutely continuous. Thus (5) holds in the ordinary sense, which yields, setting $\operatorname{Im} z=0$ in (3), the function $(A u(x)$, $u(x))$ is constant. Thus we have

$$
0 \geqslant \lim _{x \rightarrow-\infty} \sum_{j \in J_{-}} a_{j}\left|u_{j}(x)\right|^{2}=\sum_{1 \leqslant j \leqslant n} a_{j} u_{j}(x)^{2}=\lim _{x \rightarrow \infty} \sum_{j \in J_{+}} a_{j}\left|u_{j}(x)\right|^{2} \geqslant 0 .
$$

From this and (6) follows that $\hat{f}_{j}\left(-\lambda a_{j}^{-1}\right)=0$. From now, the reasoning in the proof of Lemma 4.2 of [1] is applicable.

Q.E.D.

Case (ii). Let $R(z)$ be the resolvent $(i A(d / d x)+V-z)^{-1}$ for $z \in C_{ \pm}$, $I_{+}$the injection $\left(f_{m+1}, \cdots, f_{n}\right)^{t} \rightarrow\left(0, \cdots 0, f_{m+1}, \cdots, f_{n}\right)^{t}$ and $P_{+}\left(\right.$resp. $\left.P_{o}\right)$ the 
projection $\left(f_{1}, \cdots, f_{n}\right)^{t} \rightarrow\left(f_{m+1}, \cdots, f_{n}\right)^{t}$ (resp. $\left.\left(f_{1}, \cdots, f_{m}\right)^{t}\right)$. For $z \in C_{ \pm} \quad$ we consider an operator $\tilde{L}(z)$ with domain $H_{1}(R)_{n-m}$ :

$$
\tilde{L}(z)=i \tilde{A} \frac{d}{d x}+\tilde{V}+z^{-1} W-z .
$$

First of all, note that the inverse $\tilde{R}(z)$ of $\tilde{L}(z)$ exists and that it satisfies

$$
R(z)=z^{-1}\left(-P_{o}+P_{o} V I_{+} \tilde{R}(z) P_{+}\right) \oplus I_{+} \tilde{R}(z) P_{+} .
$$

In fact, given an $f \in L^{2}(R)_{n}$, the equation $(L-z) u=f$ has a unique solution $u=R(z) f \in L^{2}(R)_{m} \oplus H_{1}(R)_{n-m}$. As one sees easily, $u=R(z) f$ if and only if

$$
\begin{aligned}
& \left(i \tilde{A} \frac{d}{d x}+\tilde{V}+z^{-1} W-z\right) P_{+} u=P_{+} f+z^{-1} P_{+} V P_{o} f, \\
& P_{o} u=z^{-1}\left(-P_{o} f+P_{o} V u\right) .
\end{aligned}
$$

Since $V_{j k}(m<j \leqslant n, 1 \leqslant k \leqslant m)$ is bounded, the range $\left(P_{+}+z^{-1} P_{+} V P_{o}\right)\left(L^{2}(R)_{n}\right)$ is equal to $L^{2}(\boldsymbol{R})_{n+m}$. Now assume that for a given $f_{+} \in L^{2}(\boldsymbol{R})_{n-m}$ the equation $\tilde{L}(z) u_{+}=f_{+}$admits two different solutions $u_{+}^{(j)}(j=1,2)$. Then, from the preceding observation, the equation $(L-z) u=I_{+} f_{+}$has two distinct solutions, which is a contradiction. The existence of $\tilde{R}(z)$ has been proved. Now (8) follows from (9). We will show that $\tilde{R}(z)$ is a $B\left(\left(L^{2, s}(R)_{n-m}\right.\right.$, $H_{1,-s}(R)$-valued continuous function on $C_{ \pm}$which has a continuous extension on $C_{ \pm} \cup R^{*}(s>1 / 2)$. To this end, note that

$$
\tilde{R}(z)+\tilde{R}_{o}(Z)\left(\tilde{V}+z^{-1} W\right) \tilde{R}(z)=\tilde{R}_{o}(z) \quad \text { for } z \in C_{ \pm},
$$

where $\tilde{R}_{o}(z)$ denotes the resolvent $(i \tilde{A}(d / d x)-z)^{-1}$. Since $\tilde{V}$ as well as $W$ belongs to $S R$ class by the assumption (**), repeating the argument in the proof of Theorem 4.2 [1], together with Lemma 3, we see that a $\boldsymbol{B}\left(H_{1,-s}(\boldsymbol{R})_{n-m}, H_{1,-s}(\boldsymbol{R})_{n-m}\right)$-valued function $\tilde{T}(z)=\tilde{R}_{o}(z)\left(\tilde{V}+z^{-1} W\right)$ has continuous extensions on $C_{ \pm} \cup R^{*}$ and that $I+\tilde{T}^{ \pm}(z)\left(z \in C_{ \pm} \cup R^{*}\right)$ is invertible if and only if $z$ is not an eigenvalue of $L$. Since $L$ has no nonzero eigenvaues, $\tilde{R}(z)$ has the boundary values $\tilde{R}^{ \pm}(\lambda)=\left(I+T^{ \pm}(\lambda)\right)^{-1} R_{o}^{ \pm}(\lambda)$, which is automatically continuous in $\lambda \in R^{*}$ :

$$
\lim _{\substack{z \rightarrow \lambda \\ \pm \operatorname{Im} z>0}} \tilde{R}(z)=R^{ \pm}(\lambda) \quad \text { in } \boldsymbol{B}\left(L^{2, s}(\boldsymbol{R})_{n-m}, H_{1,-s}(\boldsymbol{R})_{n-m}\right) .
$$

In view of (8), $R(z)$ is a $B\left(L^{2, s}(\boldsymbol{R})_{m}, L^{2, s}(\boldsymbol{R})_{m}\right) \oplus \boldsymbol{B}\left(L^{2, s}(\boldsymbol{R})_{n-m}, H_{1,-s}(\boldsymbol{R})_{n-m}\right)$-valued function which admits continuous extensions $R^{ \pm}(z)$ on $C_{ \pm} \cup R^{*}$. Now the 
absolute continuity of the spectrum of $L$ on $R^{*}$ follows.

2.3. The multiplicity of $L_{\text {ac. }}$.

Case (i). We assume the condition (*). In our case Theorem 5.1 of [1] runs as follows.

Proposition 1. There exist two families $\varphi_{ \pm}(x, \lambda)$ of generalized eigenfunctions of $L$ defined for any $\lambda \in \boldsymbol{R}$ having the following properties (recall that $L$ has no eigenvalues).

(i) As a function of $x$ and $\lambda, \varphi_{ \pm}(x, \lambda)$ is a measurable matrix valued function of class $L_{\mathrm{loc}}^{2}(\boldsymbol{R} \times \boldsymbol{R})$.

(ii) For every fixed $\lambda$ the function $\varphi_{ \pm}(x, \lambda)$ belongs to $C(R) \cap H_{1}^{\text {loc }}(R)$ and satisfies the differential equation $(i A(d / d x)+V-\lambda) \varphi_{ \pm}(x, \lambda)=0$.

(iii) For any vector $g$ in $C^{m}$, put $\varphi_{o}(x, \lambda)=e^{(i A)^{-1} x \lambda}|A|^{-1 / 2}$ and

$$
\varphi_{ \pm}^{g}(x, \lambda)=\varphi_{ \pm}(x, \lambda) g, \varphi_{o}^{g}(x, \lambda)=\varphi_{o}(x, \lambda) g .
$$

Here $|A|^{-1 / 2}$ denotes the diagonal matrix with $(j, j)$ component $\left|a_{j}\right|^{-1 / 2}$. Then for a fixed $\lambda \in \boldsymbol{R}$, the function $\varphi_{ \pm}^{g}(x, \lambda)$ has the representation

$$
\varphi_{ \pm}^{g}(x, \lambda)=\varphi_{o}^{g}(x, \lambda)-R^{\mp}(\lambda)\left[V(.) \varphi_{o}^{g}(., \lambda)\right](x),
$$

where $R^{\mp}(\lambda)$ are boundary values of the resolvent $R(z)$ of $L$. In particular $\varphi_{ \pm}^{g}(x, \lambda)$ lies in $C^{\theta,-s}(\boldsymbol{R})_{n} \cap H_{1,-s}(\boldsymbol{R})_{n}$ for any $s>1 / 2$ and satisfies the differential equation (5).

Therefore we can verify the eigenfunction expansion theorem for $L$ along the line of the proof of Theorem 6.2 [1]. Namely, define bounded linear maps $F_{ \pm}: L^{2}(R)_{n} \rightarrow L^{2}(R)_{n}$ by

$$
F_{ \pm} f(\lambda)=(2 \pi)^{-1 / 2} \lim _{N \rightarrow \infty} \int_{|x|<N} \varphi_{ \pm}^{*}(x, \lambda) f(x) d x \quad \text { in } L^{2}(\boldsymbol{R})_{n},
$$

Then $F_{ \pm}$unitarily transforms $L$ into the selfadjoint multiplication operator $M$ defined by $M f(\lambda)=\lambda f(\lambda)$.

Case (ii). We first note

Proposition 2. Let the potential $V$ be of class $C^{1}(R)$ and satisfy the first condition of the conditions (**). Then the multiplicity of $L^{\perp}$ (the restriction of $L$ to the orthogonal complement of the space $\mathfrak{S}_{0}$ spanned by eigenvectors for eigenvalue zero) is at most $n-m$.

Proof. We shall show that $L$ has an $(n-m) \times(n-m)$-matrix valued 
spectral matrix $\rho$. The proof follows the same development as that of Theorem 3.1 in Chapter 10 of [3]. However, in connection with the proof of Parseval equality we should note that the image $L\left(C_{o}^{\infty}(R)_{n}\right)$ is dense in the orthogonal complement $\mathfrak{S}_{0}^{\perp}$, that it is a subset of $D_{L}$ because $V$ is smooth and that, making use of notations in Chapter 10 [3], we have

$$
\begin{aligned}
& \int_{c<|\lambda|<1} \lambda^{2}|g(\lambda)|^{2} d \rho_{\delta} \leqslant \int_{c<|\lambda|<1}|g|^{2} d \rho_{\delta} \leqslant \int_{R}|L f(x)|^{2} d x, \\
& \int_{1<|\lambda|<\mu} \lambda^{2}|g(\lambda)|^{2} d \rho_{\delta} \leqslant \mu^{-2} \int_{1<|\lambda|<\mu} \lambda^{4}|g(\lambda)|^{2} d \rho_{\delta} \leqslant \mu^{-2} \int_{R}\left|L^{2} f(x)\right|^{2} d x .
\end{aligned}
$$

The lemma below completes the proof of our theorem.

LEMMA 4. Let $L_{o}$ be the selfadjoint operator $i A(d / d x)$ in $L(R)_{n}$. For any $f \in C_{o}^{\infty}(R)_{n}$ of the form $f=\left(0, \cdots, 0, f_{m+1}, \cdots, f_{n}\right)^{t}, e^{i t L} e^{-i t L_{o}} f$ converges strongly as $t \rightarrow \infty$.

Proof. As is well known ([5], Theorem 3.7 in Chapter $X$ ), the convergence follows from the fact that $\left\|V e^{-i t L_{0}} f\right\|$ is integrable on some interval $\left(t_{o}, \infty\right)$. By the assumption $\left({ }^{* *}\right)$ there exist positive constants $\varepsilon, K$ and $r(>1)$ such that $\left|V_{j k}(x)\right| \leqslant K|x|^{-1-s}$ for $|x|>r$. Since $\left(e^{-i t L_{o}} f\right)_{j}(x)=$ $f_{j}\left(x+a_{j} t\right)$, assuming that a finite interval $(-c, c)$ includes the support of $f$ and denoting $\min _{m<j}\left|a_{j}\right|$ (resp. $\left.\sup _{j, x}\left|f_{j}(x)\right|\right)$ by a (resp. s), we have the following inequality:

$$
\left\|V e^{-i t L_{o}} f\right\|^{2} \leqslant 2 c K^{2} s^{2} n^{3}|c+a t|^{-2-2 s},
$$

which yields the desired integrability of $\left\|V e^{-i t L_{o}} f\right\|$.

Q.E.D.

Proposition 2 and Lemma 4 imply that $L_{\mathrm{ac}}$ is unitary equivalent to the multiplication operator in $L^{2}(R)_{n-m}$. Since we have shown that $L_{\mathrm{c}}=$ $L_{\text {ac }}$ (see 2.2), the last assertion of our theorem has been proved.

\section{§3. Sufficient condition for $L$ to have no eigenvalues}

As stated in $\S 1, A$ denotes a real diagonal matrix, while $V$ stands for an Hermitian matrix valued function of class $L_{\text {loc }}^{2}(R)$.

Proposition 3. (i) Assume that $\operatorname{det} A \neq 0$. If $A$ is positive (or negative) definite or if $V$ is integrable on a half line, then $L$ has no eigenvalue.

(ii) Assume that $a_{1}=\cdots=a_{m}=0$ and $a_{m+1} \cdots a_{n} \neq 0$ for some $0<$ $m<n$ and that 


$$
\begin{aligned}
& V_{j k}=0 \quad \text { for } 1 \leqslant j, k \leqslant m, \\
& V_{j k} \text { and } W_{j k}=\sum_{1 \leq i \leq m} V_{j \ell} V_{\ell k} \text { are integrable on a common half line } \\
& \text { for } m<j, k \leq n,
\end{aligned}
$$

then $L$ has no eigenvalues differing from zero.

Proof. Suppose $u \in D_{L}$ satisfies the following equation for a real $\lambda$.

$$
\left(i A \frac{d}{d x}+V-\lambda\right) u=0 \text {. }
$$

We shall show that $u=0$. Note that $u_{j}$ are absolutely continuous in the case (i) and that $u_{j}(j>m)$ are also absolutely continuous in the case (ii) (cf. the proof of Lemma 1). If $A$ is definite, (3) implies that the function $(A u(x), u(x))$ is constant, thus $u=0$. If $V$ is integrable, say on $(0, \infty)$, define $v \in L^{2}(R)_{n}$ by the formula $u=e^{(i A)-1 x \lambda} v$. Then $v$ satisfies

$$
v^{\prime}(x)=e^{-(i A)^{-1} x^{\lambda}} V(x) e^{(i A)^{-1} x \lambda} v(x) .
$$

Since $v$ has a non-zero limit as $x \rightarrow \infty$, provided $v \neq 0$ ([3], problem 6 in Chapter 3), we conclude that $u=0$. In the case (ii) we must show $u=0$, assuming that $\lambda \neq 0$. We rewrite (11) in the form (9) with $f=0$ and $z=\lambda$. Since the Hermitian matrix valued function $\tilde{V}+\lambda^{-1} W$ is integrable on a half line, it follows that $P_{+} u=0$ via the same reasoning for the case (i). From the second equality of (9), $P_{o} u=0$. Thus $u=0$.

Q.E.D.

\section{REFERENCES}

[1] S. Agmon, Spectral properties of Schrodinger operators and scattering theory, Annali della Scuol Normal Superiore di Pisa, series 4, vol. 2 (1975), 51-218.

[2] E. Angelopoulos, Reduction on the Lorentz subgroup of UIR's of the Poincaré group induced by a semisimple little group, Math. Phys. vol. 15 (1974), 155-165.

[ 3 ] E. A. Coddington, N. Levinson, Theory of ordinary differential equations, McGraw-Hill, 1955.

[4] H. Kaneta, Irreducibility of some unitary representations of the Poincaré group with respect to the Poincaré subsemigroup, I, Nagoya Math. J. vol. 78 (1980), 113-136.

[ 5] T. Kato, Perturbation theory for linear operators, Springer, 1966.

[6] V. V. Martynov, Conditions for discreteness and continuity of the spectrum of a selfadjoint operator of first order differential equations, Dokl. Acad. Nauk, SSSR, 165 (1965), 986-991.

[ 7 ] K. Mochizuki, Spectral and scattering theory for symmetric hyperbolic system in an exterior domain, Pub. RIMS, Kyoto Univ., 5 (1969), 219-258.

[8] K. Yajima, The limiting absorption principle for uniformly propagative systems, J. Fac. Sci. Univ. Tokyo Sec. 1A, 21 (1974), 119-131. Eigenfunction expansions associated with uniformly propagative systems and their applications to scattering theory, J. Fac. Sci. Univ. Tokyo, Sec. 1A, 22 (1975), 121-151. 
Department of Mathematics Faculty of Science

University of Shizuoka

Department of Mathematics

Faculty of Science

University of Nagoya 